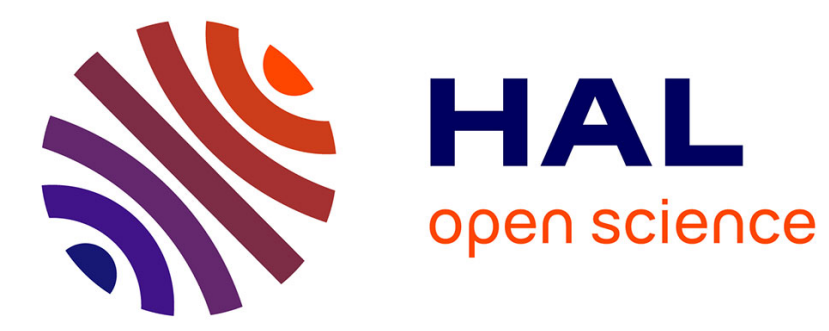

\title{
Experimental study of low inertia vortex rings in shear-thinning fluids
}

Omar Bentata, Dominique Anne-Archard, Pierre Brancher

\section{To cite this version:}

Omar Bentata, Dominique Anne-Archard, Pierre Brancher. Experimental study of low inertia vortex rings in shear-thinning fluids. Physics of Fluids, 2018, 30 (11), pp.1-10. 10.1063/1.5048841 . hal01958621

\section{HAL Id: hal-01958621 \\ https://hal.science/hal-01958621}

Submitted on 18 Dec 2018

HAL is a multi-disciplinary open access archive for the deposit and dissemination of scientific research documents, whether they are published or not. The documents may come from teaching and research institutions in France or abroad, or from public or private research centers.
L'archive ouverte pluridisciplinaire HAL, est destinée au dépôt et à la diffusion de documents scientifiques de niveau recherche, publiés ou non, émanant des établissements d'enseignement et de recherche français ou étrangers, des laboratoires publics ou privés. 


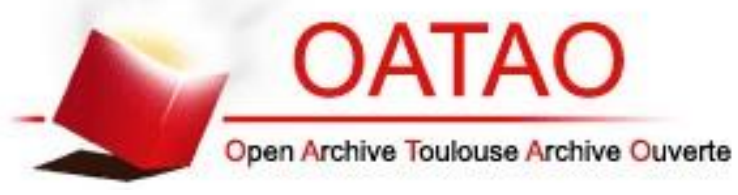

\section{Open Archive Toulouse Archive Ouverte}

OATAO is an open access repository that collects the work of Toulouse researchers and makes it freely available over the web where possible

This is an author's version published in: http://oatao.univ-toulouse.fr/21314

\section{Official URL:}

https://doi.org/10.1063/1.5048841

\section{To cite this version:}

Bentata, Omar and Anne-Archard, Dominique and Brancher, Pierre Experimental study of low inertia vortex rings in shearthinning fluids. (2018) Physics of Fluids, 30 (113103). 1-10. ISSN 1070-6631

Any correspondence concerning this service should be sent to the repository administrator: tech-oatao@listes-diff.inp-toulouse.fr 


\title{
Experimental study of low inertia vortex rings in shear-thinning fluids
}

\author{
O. Bentata, ${ }^{1}$ D. Anne-Archard, ${ }^{2}$ and P. Brancher ${ }^{2}$ \\ ${ }^{1}$ Laboratory of Aeronautic and Propulsive Systems (LAPS), Université des Sciences et de la Technologie d'Oran \\ Mohamed Boudiaf, USTO-MB, BP 1505, El M'Naouar, 31000 Oran, Algeria \\ ${ }^{2}$ Institut de Mécanique des Fluides de Toulouse (IMFT), Université de Toulouse, CNRS, Toulouse, France
}

\begin{abstract}
The present work investigates experimentally the dynamics of vortex rings in shear-thinning fluids at low generalized Reynolds numbers, with a focus on the range from 300 down to 30 . The experimental apparatus consists of a vertical cylinder-piston system with the lower part immersed in a tank filled with the liquid. Particle image velocimetry is used to analyze the influence of the non-Newtonian nature of the fluid on the generation, propagation, and eventual dissipation of vortex rings. The results show that shear-thinning controls the generation phase, whereas the vortex ring subsequent evolution is independent of the power-law index. In particular, it is found that the final dissipation stage is characterized by a flow dynamics which tends ultimately to a regime at a constant viscosity corresponding to the Newtonian plateau. This reveals the role of the Carreau number and of the Reynolds number based on this specific viscosity as relevant control parameters for this last stage.
\end{abstract}

https://doi.org/10.1063/1.5048841

\section{INTRODUCTION}

Vortex rings are ubiquitous coherent structures that are observed in numerous industrial, biological, and natural phenomena. Many examples can be found in nature, like in the wake of birds and fishes, ${ }^{1}$ or in the human heart following the discharge of blood into the ventricles. ${ }^{2}$ Vortex rings are known to propagate by self-induction, as shown by the smoke rings expelled by experienced smokers, and to transport mass, momentum, and heat. Therefore, they are of fundamental importance in specific industrial contexts. They are produced by moving blades in industrial mixing systems, 3,4 or in internal combustion engines and injection systems. ${ }^{5}$ It is most likely that all the potentialities of vortex rings have not been explored yet and new applications are appearing regularly (see, for instance, An et al. ${ }^{6}$ for the freezing of vortex rings and the possible use of this process for bioencapsulation).

At the laboratory scale, vortex rings can be generated experimentally by different techniques. The most common method is the impulsive ejection of a volume of fluid by a piston-cylinder device into a quiescent environment, a technique that has been widely used in the last decades. ${ }^{7-10}$ The translation of the piston in the cylinder during a finite time produces an impulsive jet flow whose shear layer vorticity rolls up into a toroidal structure to form a vortex ring. During this formation phase, the piston diameter, the piston velocity, and the length of the piston stroke control the development of the boundary layer within the tube, the vorticity profile at the exit, and therefore the structure of the vortex ring. When inertia dominates, the vortex ring detaches itself from the tube exit and propagates under the influence of its self-induced velocity field. In this post-formation phase, the vortex ring slowly thickens by viscous diffusion and entrainment of ambient fluid before an eventual transition to turbulence at large Reynolds numbers.
The dynamics of vortex rings have been extensively studied, starting from the pioneering work of Helmholtz, ${ }^{11}$ Kelvin, ${ }^{12}$ and Thomson ${ }^{13}$ in the second half of the nineteenth century. Many studies have focused on the characterization of the vortex ring structure, in terms of diameter, circulation, core size, and propagation speed, depending on the operating conditions of vortex ring generation. ${ }^{8,14-21}$ It has been shown that successive vortex rings are produced for large piston stroke to diameter $\left(L_{p} / D_{p}\right)$ ratios, according to a mechanism related to the Kelvin-Helmholtz instability of the jet exiting from the tube, whereas small stroke ratios generate a single vortex ring. ${ }^{22}$ The stability of vortex rings has been an axis of active research over the past forty years. ${ }^{7,9,10,23-25}$ The instability of vortex rings is related to the three-dimensional elliptic instability which is activated in vortices subjected to a strain field and characterized by an azimuthal deformation of the ring. In particular, it has been observed that vortex rings were stable for Reynolds numbers $R e=\rho U D / \eta<600$, where $U$ and $D$ denote the propagation speed and the diameter of the ring, respectively, with $\eta$ being the dynamic viscosity of the fluid. ${ }^{7}$ The extreme varieties of issues discussed in the study of vortex rings is summarized by Saffman: ${ }^{26}$ "The vortex ring is a complete problem of fluid mechanics" (see also the recent reviews by Meleshko et al. ${ }^{27}$ and Velasco Fuentes ${ }^{28}$ for an historical and artistic perspective, respectively).

Despite this rich literature, only few studies have been carried out on vortex rings in non-Newtonian fluids, with the notable exception of the experiments by Palacios-Morales and Zenit, ${ }^{29}$ who studied both the influence of shear-thinning (power-law index $n$ ) and of the piston stroke-to-diameter ratio $L_{p} / D_{p}$. The study was conducted for a generalized Reynolds number in the range 140-600 (or 200-1000 with our definition for the Reynolds number, see Sec. II), and the authors found that the vortex circulation decreases with the power 
index $n$. The authors observed that while the initial diameter of the vortex ring does not depend on the rheological properties, shear-thinning vortices tend to expand in the radial direction after some discharge time, and the travel velocity is reduced.

Most of the other studies concerned with vortices in nonNewtonian fluids are devoted to the different regimes in the flow past a cylinder. These studies focused on how shearthinning properties affect the transition Reynolds numbers between these regimes. In order to study the influence of elasticity while taking into account a shear-thinning behavior, Coelho and Pinho ${ }^{30}$ showed that a generalized Reynolds number $R e^{*}$ could be defined based on the viscosity at a characteristic shear rate such that its value at the onset of laminar vortex shedding matches the Newtonian one. Chandra and Chhabra $^{31}$ studied the flow over a semi-circular cylinder. They showed that a shear-thinning behavior delayed the onset of the laminar vortex shedding regime, but they also brought out a complex role of the power-law index with a non-monotonic evolution of the critical Reynolds numbers. The same was observed by Rao et al. ${ }^{32}$ in the case of a confined circular cylinder. Sahu et al. examined the same issue for a square cylinder in an infinite medium ${ }^{33}$ and in a confined domain. ${ }^{34}$ Vortices are also observed in the flow through a sudden expansion. In this case, the shear-thinning properties delay the onset of the different regimes (vortex formation, asymmetry, etc.) with a relatively monotonic influence of the power-law index. ${ }^{35,36}$ Another example is the study of a hairpin vortex in a wallbounded flow by Zhen et al., ${ }^{37}$ for a Reynolds number range (4000-8000) higher than for the present study. The authors observed that shear-thinning properties decreased the production of vortex kinetic energy, increased the viscous dissipation, and delayed the transition to a turbulent state.

In this context, the aim of the present study is to provide with a better understanding of the global dynamics of vortex rings in shear-thinning fluids at low Reynolds numbers. More particularly, the present work focuses on the generation, detachment, and subsequent evolution of a single vortex ring for generalized Reynolds numbers between 30 and 300. Particle Image Velocimetry (PIV) has been used to get qualitative and quantitative results on the flow dynamics. Section II describes the experimental setup and the measurement techniques. Experimental results are shown and analyzed in Sec. III. Conclusions are presented in Sec. IV.

\section{EXPERIMENTAL SETUP AND MEASUREMENT TECHNIQUES}

A schematic diagram of the experimental device is presented in Fig. 1. The vortex rings were generated using a piston-cylinder system. The cylinder was vertical and immersed in the fluid down to a depth of $4 \times D_{p}$, where $D_{p}$ is the internal diameter of the cylinder. The piston was entrained by a step motor driven by a computer. Vortex rings were produced by translating the piston from a height $Z_{i}$ to a height $Z_{f}$ (corresponding to a stroke length $L=Z_{f}-Z_{i}$ ) with a given velocity $V_{p}$. Acceleration and deceleration phases were short enough so that $V_{p}$ could be considered as constant during the displacement. The minimum time interval between two experiments was fixed to at least $20 \mathrm{~min}$ in order to let the fluid come to rest before the start of a new sequence. In this study, the final position of the piston is chosen to be at the exit section of the cylinder. The vertically downward $z$-axis coincides with the centerline of the cylinder, and the nozzle exit plane marks the origin $z=0$.

The internal and external diameters of the cylinder are $D_{p}=21 \mathrm{~mm}$ and $25 \mathrm{~mm}$, respectively. The immersed end of the tube was shaped to form a wedge with a tip angle $\theta=45^{\circ}$ on the outer wall (Fig. 2). The dimensions of the tank were $L \times W \times H=24 \times 24 \times 60 \mathrm{~cm}^{3}$. Solutions of xanthan gum (G1253, Sigma-Aldrich) were used as shear-thinning fluids. They were prepared by dispersing the polymer in distilled water and then left under mild stirring for $24 \mathrm{~h}$ before use. Two concentrations were used: $0.02 \%$ (X1) and $0.04 \%$ (X2). Rheological characterisations were conducted on a Mars III rheometer (Thermo Sc.) equipped with a cone and plate geometry (60 $\mathrm{mm}$ diameter, $2^{\circ}$ angle). Xanthan solutions exhibit nonNewtonian shear-thinning behavior with a Newtonian plateau for the low shear rates. Measurements of elastic $\left(G^{\prime}\right)$ and viscous $\left(G^{\prime \prime}\right)$ modulus confirmed that elasticity was negligible for both solutions: the ratio of $G^{\prime}$ to $G^{\prime \prime}$ was 0.1 at $0.01 \mathrm{~Hz}$ and 0.46 at $1 \mathrm{~Hz}$ for $\mathrm{X} 2$ fluid. No measurements were possible beyond $1 \mathrm{~Hz}$ because of too strong inertia effects in these low-viscosity fluids.

A Carreau-Yasuda model was fitted on the dynamic viscosity data, ${ }^{38}$

$$
\eta=\eta_{0}\left[1+(\lambda \dot{\gamma})^{a}\right]^{\frac{n-1}{a}}
$$

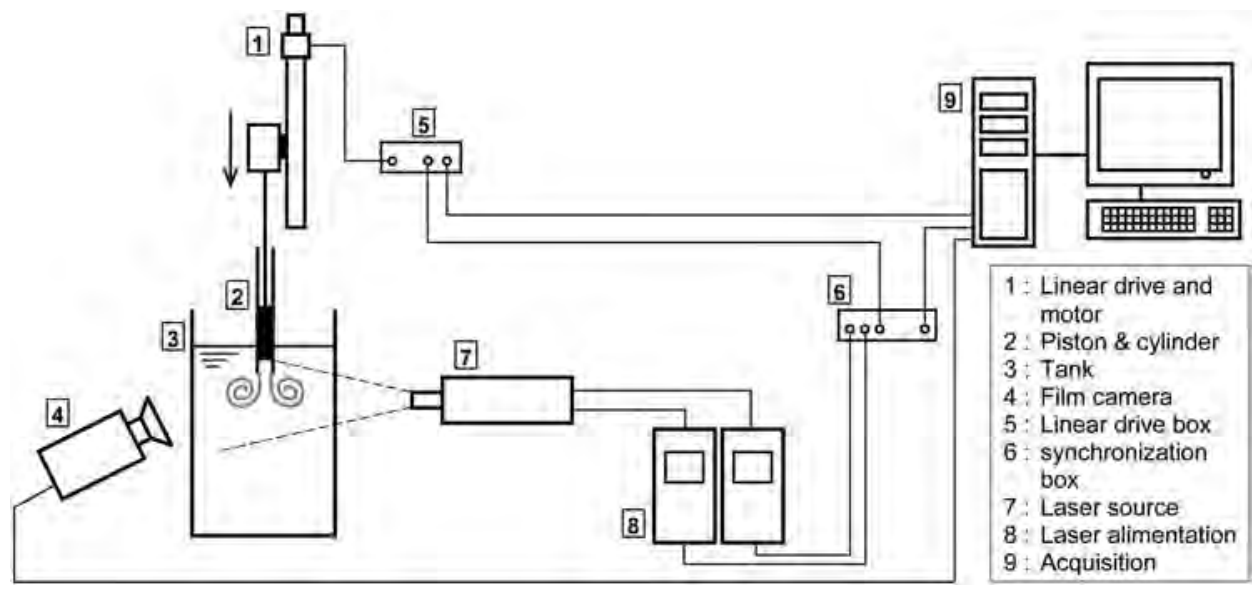

FIG. 1. Sketch of the experimental setup. 


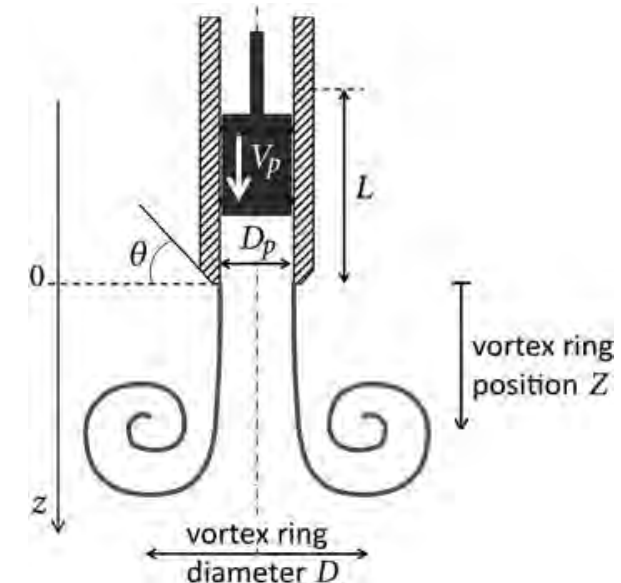

FIG. 2. Piston-cylinder system for vortex ring generation.

where $\eta_{0}$ is the dynamic viscosity of the Newtonian plateau and $n$ is the classical power-law index for the shear-thinning region. Parameters were adjusted on the shear rate range $\left[0.01 \mathrm{~s}^{-1} ; 300 \mathrm{~s}^{-1}\right]$, and values are reported in Table I. Also reported are the quantities $k=\eta_{0} \lambda^{n-1}$ and $\dot{\gamma}_{0}=1 / \lambda$ which are the consistency (power-law region) and the shear rate for transition between Newtonian and shear-thinning behaviors, respectively.

The two xanthan solutions exhibit a Newtonian plateau for low shear rates and a shear-thinning behavior for higher ones. The transition occurs for the critical shear rate $\dot{\gamma}_{0}=1 / \lambda$, where $\lambda$ can be interpreted as a characteristic time of the fluid. The Carreau number $C r$ is defined as the product of $\lambda$ by a characteristic shear rate of the flow. ${ }^{39,40}$ Using the wall shear rate in the cylinder for a power-law fluid, ${ }^{41}$ the Carreau number writes

$$
C r=\lambda\left(\frac{3 n+1}{n}\right) \frac{2 V_{p}}{D_{p}} .
$$

Note that the Newtonian case corresponds to $C r=0 . C r$ values are reported in Table II. It can be noticed that the wall shear rate during injection is expected to be higher than the value used for the $\mathrm{Cr}$ calculation since the profile in the cylinder is not established (see Sec. III A). Therefore, the Carreau number is systematically underestimated. Since $\mathrm{Cr}$ is significantly higher than 1 , even in the case $R e=30$, it can be affirmed that the fluid is actually characterized by a shear-thinning behavior during the injection phase for all the cases studied here.

TABLE I. Rheological parameters of the test liquids.

\begin{tabular}{llllccc}
\hline \hline Fluids (\%) & $\eta_{0}(\mathrm{~Pa} \mathrm{~s})$ & $n(-)$ & $\lambda(\mathrm{s})$ & $a(-)$ & $\dot{\gamma}_{0}\left(\mathrm{~s}^{-1}\right)$ & $k\left(\mathrm{~Pa} \mathrm{~s}^{-n}\right)$ \\
\hline $\mathrm{X} 1(0.02)$ & 0.0081 & 0.76 & 2.1 & 3 & 0.48 & 0.0068 \\
$\mathrm{X} 2(0.04)$ & 0.023 & 0.65 & 2.06 & 1.1 & 0.49 & 0.0178 \\
\hline
\end{tabular}

TABLE II. Values of the Carreau number for the present parametric study.

\begin{tabular}{lccccc}
\hline \hline $\operatorname{Re}$ & 30 & 60 & 90 & 200 & 300 \\
\hline $\mathrm{X} 1(0.02 \%)$ & 7.4 & 12 & 19 & 32 & 42 \\
$\mathrm{X} 2(0.04 \%)$ & 13 & 22 & 30 & 61 & 81 \\
\hline \hline
\end{tabular}

A mineral oil (Marcol 82, Exxon Mobil) was used as a Newtonian reference fluid for comparison (with a viscosity of $0.03 \mathrm{~Pa} \mathrm{~s}$ at $20^{\circ} \mathrm{C}$ ). Experiments were performed at room temperature, and the viscosity changes were compensated by the adjustment of the piston velocity.

For the Newtonian case, the Reynolds number $R e$ $=\rho V_{p} D_{p} / \eta$ is defined from the operational conditions for the piston in the cylinder, where $\eta$ is the dynamic viscosity of the fluid. A generalized Reynolds number is used for shear-thinning fluids, which is based on the extension to powerlaw fluids of the classical friction rule in cylindrical tubes $f=16 / \operatorname{Re},^{41}$

$$
\operatorname{Re}_{g}=8^{1-n}\left(\frac{4 n}{3 n+1}\right)^{n} \frac{\rho V_{p}^{2-n} D_{p}^{n}}{k} .
$$

The choice of this generalized Reynolds number as a control parameter of the vortex ring generation process is consistent with the previous observation that the fluid has a shearthinning behavior during the injection phase (Carreau number larger than unity for all the cases studied here). The stroketo-diameter ratio $L_{p} / D_{p}$ was set to 3 , which is an intermediate value sufficient to generate a vortex ring while ensuring that only one vortex ring is produced. ${ }^{22,42}$ Lengths and velocities are nondimensionalized by $D_{p}$ and $V_{p}$, respectively. The dimensionless quantities are hereafter identified by a superscript ${ }^{*}$. Time is consequently non-dimensionalized by $D_{p} / V_{p}$. Therefore, $t^{*}=L_{p} / D_{p}=3$ corresponds to the stopping time of the piston, which starts moving at $t^{*}=0$.

A Particle Image Velocimetry (PIV) technique was used to determine the velocity field. The PIV system was composed of a CCD camera (PCO 2000, $2048 \times 2048$ pixels) and a Nd-YAG laser (Quantel Twin CFR 200, 200 mJ) generating a laser sheet. The laser and camera were controlled via a synchronizing unit, designed in the laboratory, which receives a trigger signal from the piston when it starts. The laser sheet was carefully positioned in a vertical diametrical plane of the cylinder and orthogonal to the tank wall. The camera axis was horizontal and perpendicular to the laser sheet. The fluid was seeded with Orgasol polyamide spheres of $60 \mu \mathrm{m}$ diameter. Velocity fields were computed using the PIVIS software developed in the laboratory. ${ }^{43}$ The algorithm is based on a 2D FFT cross-correlation function implemented in an iterative subpixel interpolation scheme. The flow was analyzed by cross-correlating $50 \%$ overlapping windows of $32 \times 32$ pixels. This procedure yields velocity field of $127 \times 127$ vectors and a spatial resolution of $0.64 \mathrm{~mm}$, based on the cross-correlation window size. Data were analyzed to check that no peak-locking phenomenon was present.

The velocity field quality was improved by performing the two following operations: (i) a preprocessing of the images before PIV calculation (correction of gray levels and subtraction of the local average level of gray) and (ii) an averaging of the velocity fields obtained for five realizations of the same sequence. Moreover, false vectors were identified by comparing average and instantaneous velocity fields, and they were removed for a second averaging stage.

PIV measurements provided the radial $\left(V_{r}\right)$ and vertical (or axial, $V_{z}$ ) components of the velocity field. The azimuthal velocity component $V_{\theta}$ was zero because of the rotational 
symmetry. These data were used to compute the azimuthal vorticity $\omega=V_{r, z}-V_{z, r}$. The shear rate $\dot{\gamma}$ was computed from the components of the strain rate tensor $\mathbb{D}=\frac{1}{2}\left(\nabla \mathbf{V}+\nabla \mathbf{V}^{T}\right)$. Its general form is $\dot{\gamma}=\left[2 \operatorname{tr}\left(\mathbb{D}^{2}\right)\right]^{1 / 2}$, where $\operatorname{tr}$ denotes the trace. Assuming axisymmetry, the computation of the shear rate in a plane containing the axis of symmetry requires only the radial and axial components of the velocity field, $\dot{\gamma}=\left[2 V_{r, r}^{2}+2 V_{r}^{2} / r^{2}+2 V_{z, z}^{2}+\left(V_{r, z}+V_{z, r}\right)^{2}\right]^{1 / 2}$.

The vortex ring position was defined by the vertical location $Z^{*}(t)$ of the point where the axial velocity on the axis $V_{z}^{*}\left(r^{*}=0, z^{*}\right)$ is maximum. Then one can define a relative frame of reference based on the propagation speed of the vortex ring. In this relative frame of reference, the vortex ring diameter $D$ was measured as the distance between the two stagnation points at the center of the closed instantaneous streamlines around the vortex ring core. It is noteworthy that this characterization method exclusively based on the velocity field avoids the classical problems associated with the post-processing of the vorticity field, whose signal-to-noise ratio usually makes difficult the detection of the vortex core center in a robust and objective way.

\section{RESULTS}

\section{A. Velocity at the cylinder exit during the vortex ring generation}

The time evolution of the axial velocity profiles near the cylinder exit $\left(z^{*}=0.1\right)$ is presented in Fig. 3 for a vortex ring generated in the X2 fluid. When the piston starts, the velocity profile is flat $\left(t^{*}=0.93\right)$ and then progressively tends toward a Poiseuille profile. The theoretical maximum value of axial velocity corresponding to the exact steady solution for the present fluid ( $n=0.65$ ) is equal to 1.79 . This theoretical value is almost reached at $t^{*}=1.87$. As the piston approaches the exit section, the trend is reversed and the jet flow at the exit tends toward a top-hat velocity profile $\left(t^{*}=2.58\right.$ and 2.81$)$. Once the piston has stopped $\left(t^{*}>3\right)$, the velocity profile quickly goes to zero. Outside the cylinder $\left(r^{*}>0.5\right)$, a counter-flow can be observed at the beginning of the piston movement $\left(V_{z}^{*}<0\right.$ for

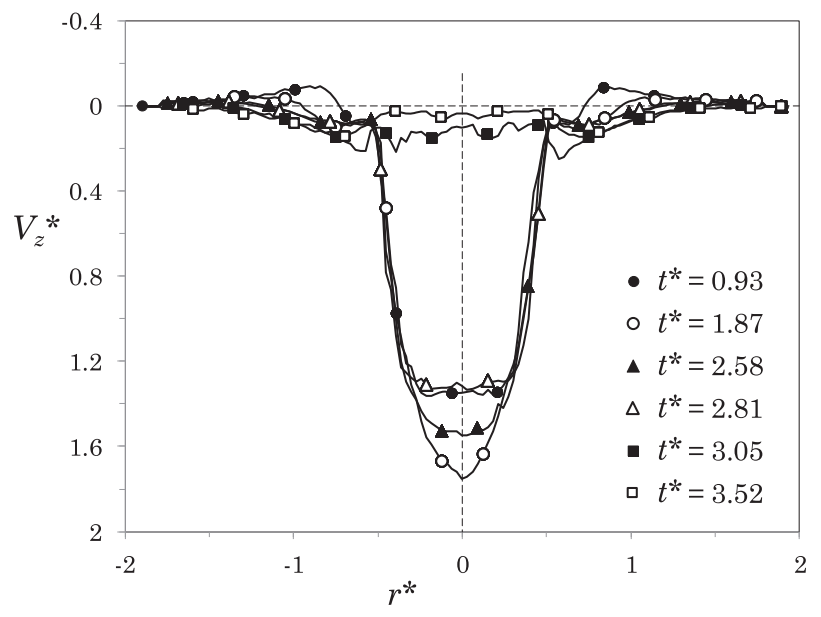

FIG. 3. Time evolution of the axial velocity profile near the cylinder exit $\left(Z^{*}=0.1\right)$ for fluid X2 $(n=0.65)$ at $R e_{g}=90$. (Note that the symbols do not mark the data points but are only used to distinguish between the different curves.)

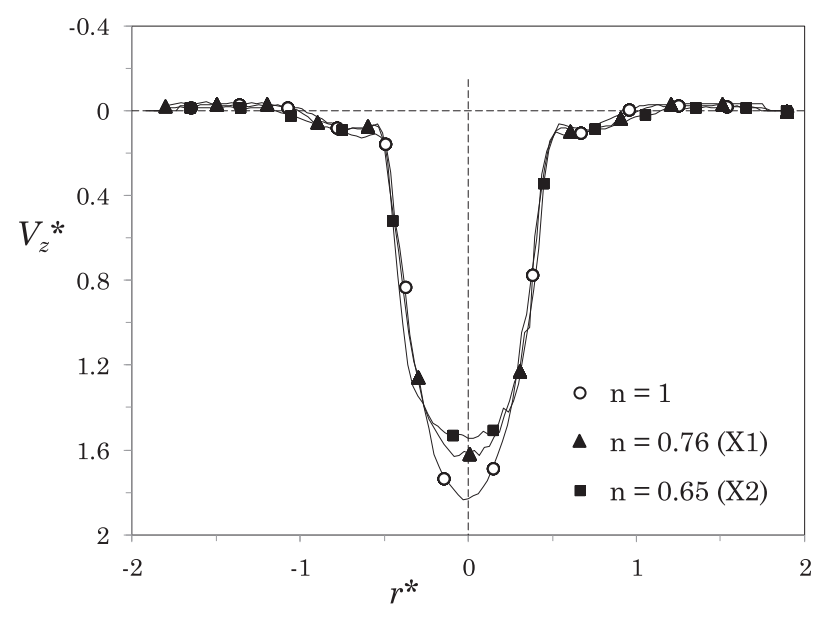

FIG. 4. Influence of the power-law index $n$ on the axial velocity profile near the cylinder exit at a given time $t^{\star} \simeq 2.56 \pm 5 \%\left(R e=R e_{g}=90\right)$. (Note that the symbols do not mark the data points but are only used to distinguish between the different curves.)

$\left.t^{*}=0.93\right)$. This phenomenon is reversed at later times, and the entrainment of fluid at the cylinder periphery is then observed in the jet direction. It is noteworthy that the velocity profiles at a given time overlap as expected for the different Reynolds numbers in the range studied here (data not shown).

The influence of shear-thinning is illustrated in Fig. 4, which presents axial velocity profiles for two different shearthinning fluids $\mathrm{X} 1(n=0.76)$ and $\mathrm{X} 2(n=0.65)$ and for the Newtonian reference case. As expected, the velocity profiles are flatter as $n$ decreases. It can be noted that the power-law index $n$ has a greater influence on this unsteady situation than on the steady Poiseuille case: the maximum values for the $V_{z}^{(n)} / V_{z}^{(n=1)}$ ratio are 0.866 and 0.845 for $n=0.76$ and 0.65 , respectively, in the present case, to be compared to 0.93 and 0.895 for the theoretical steady Poiseuille profiles. $^{41}$

\section{B. Vorticity field and instantaneous streamlines}

The time evolution of the vorticity field associated with the generation and propagation of the vortex ring is presented in Figs. 5 and 6 for the Newtonian reference case and for the X1 fluid, respectively. The instantaneous streamlines in the frame of reference moving with the vortex ring are superimposed to the vorticity field for $t^{*}>3$ (once the vortex ring has detached from the cylinder exit). Vorticity computation being based on velocity gradients, experimental errors from the PIV measurements are exacerbated, but we chose not to smooth out (nor symmetrize) the fields. This allows the level of error to be at least approximately inferred from the figures.

The observation window in Figs. 5 and 6 is the same for the different cases, namely, between $z^{*}=0$ and $z^{*}=3.5$ and between $r^{*}=-1.75$ and $r^{*}=+1.75$. For both Newtonian and shear-thinning fluids, one can observe the formation of an axisymmetric coherent structure associated with the ejection and roll-up of the vortex sheet generated at the boundary layer on the inner side of the cylinder. It is noteworthy that no azimuthal instability has been observed for the present range of Reynolds numbers, regardless of the nature of the fluid (in a Newtonian fluid, such instabilities are actually expected to 
(a) $t^{*}=2.6$
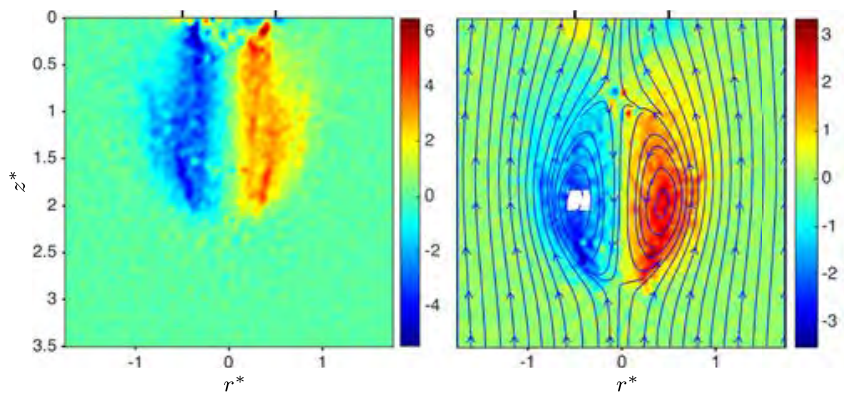

be inactive for $R e<600^{7}$ ). Therefore, the vortex rings are expected to be intrinsically axisymmetric, at least within experimental errors. This point can be checked in Figs. 3-6 that display both parts of the symmetry plane. The observed symmetry is not a full guarantee of axisymmetry, but it gives some reasonable confidence in the structural axisymmetry of the flow. (c) $t^{*}=6.4$

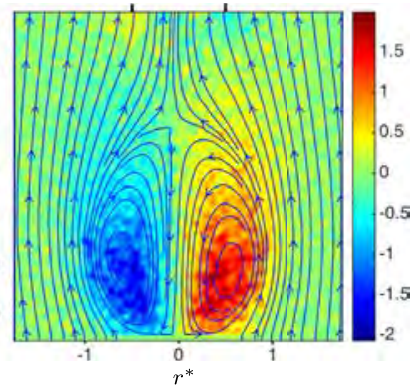

FIG. 5. Time evolution of vorticity field and instantaneous streamlines in the vortex ring frame of reference for the Newtonian case $(R e=60)$.

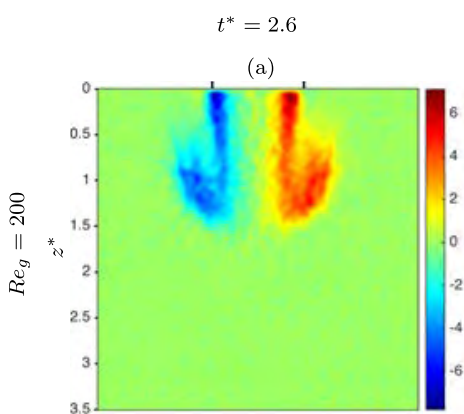

(d)

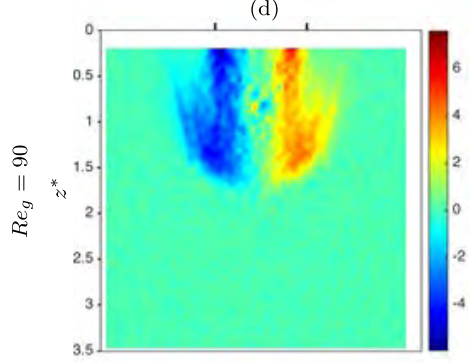

(g)
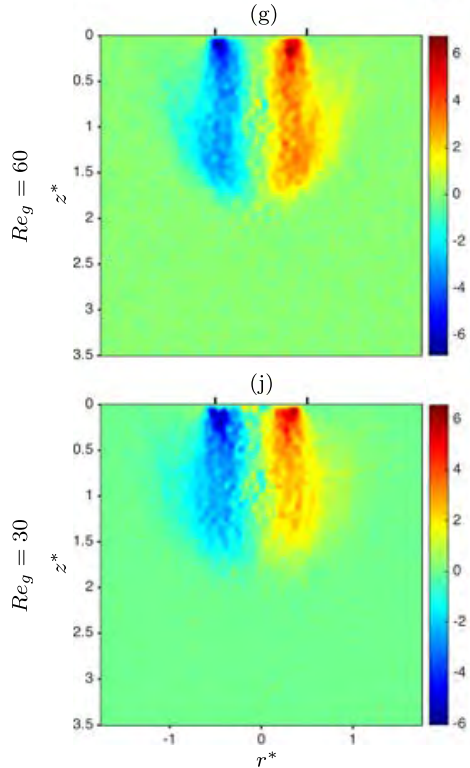

$t^{*}=4.5$

(b)

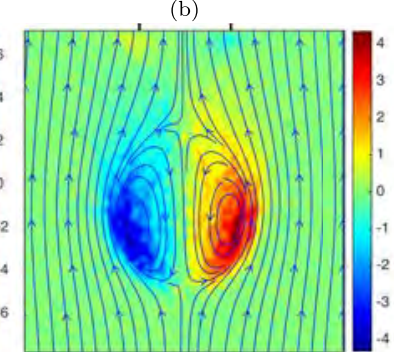

(e)

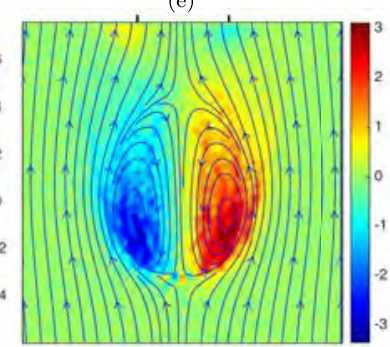

(h)

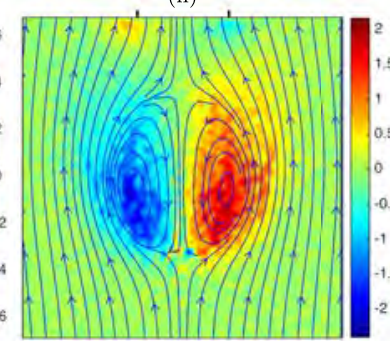

(k)

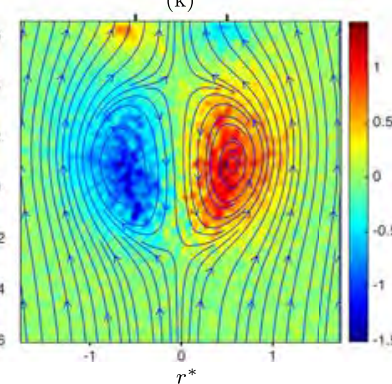

In the Newtonian case at $R e=60$ (Fig. 5), the vortex ring propagates across the observation windows at a constant speed. The same behavior is observed for the shear-thinning fluid at generalized Reynolds numbers from 200 down to 90 (Fig. 6). By contrast, the scenario is different at lower Reynolds numbers in the shear thinning case $\left(R e_{g}=60\right.$ and $R e_{g}=30$, Fig. 6): the vortex ring slows down and eventually stops while

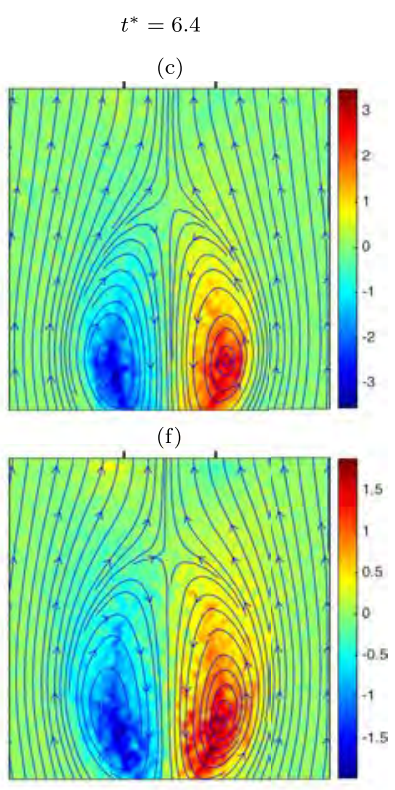

(i)

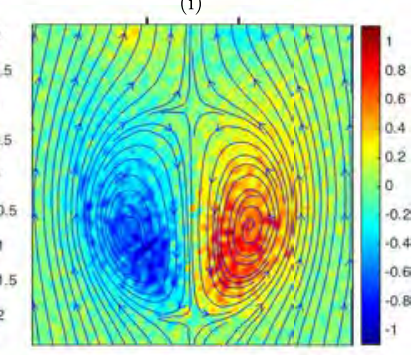

(1)

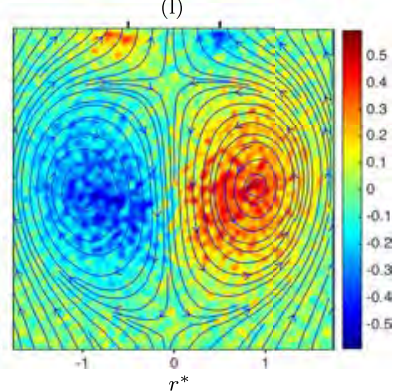

FIG. 6. Time evolution of vorticity field and instantaneous streamlines in the vortex ring frame of reference for the $\mathrm{X} 1$ fluid $(n=0.76)$ at different generalized Reynolds numbers. 


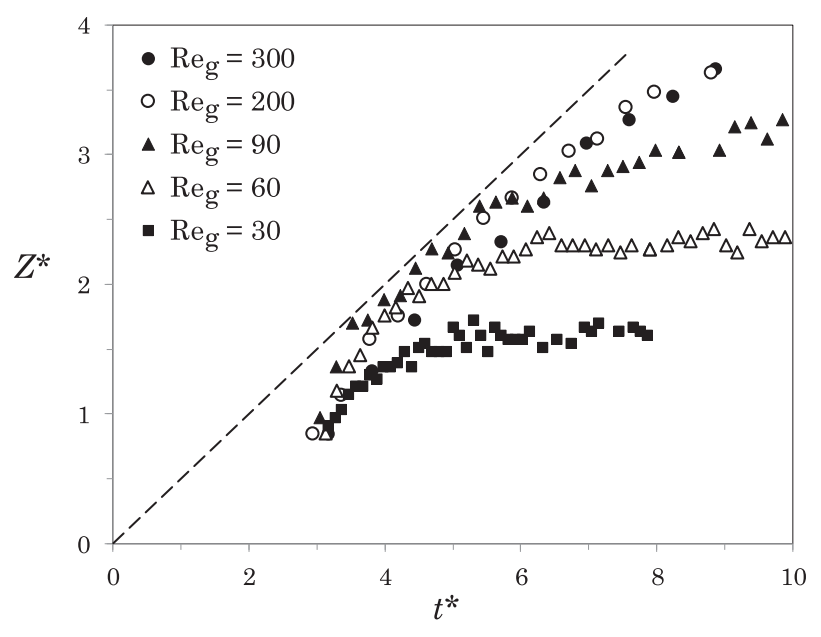

FIG. 7. Time evolution of the vortex ring position in the X2 fluid $(n=0.65)$ for different Reynolds numbers.

its diameter increases. These points are examined in detail in the next sections.

\section{Vortex ring propagation}

Figure 7 presents the time evolution of the vortex ring vertical position $Z^{*}$ for the $\mathrm{X} 2$ fluid $(n=0.65)$ and for generalized Reynolds numbers from 300 down to 30 . The vortex ring position has been observed to evolve monotonously in the present range of Reynolds numbers. In a first stage, the propagation is similar for all $R e_{g}$ and coincides with the piston velocity (slope 1 with the present scaling). For the largest Reynolds numbers (200 and 300), the vortex ring later reaches a quasi-constant propagation speed, close to half the piston velocity (dashed line with slope 1/2 in Fig. 7). As for Newtonian vortex rings, this velocity is controlled by the inertial scales, namely, the piston diameter and velocity, and is therefore expected to be independent of the Reynolds number in the inertial regime. This is different when the flow approaches the viscous regime, as seen in the present study for Reynolds number 90 and lower. The vortex ring progressively slows down before it eventually stops at a distance which decreases as the Reynolds number is lowered.

The influence of the rheological nature of the fluid on this dynamics at low Reynolds numbers is illustrated in Fig. 8. At $R e=90$ [Fig. 8(a)], the first stages are similar for all fluids. During the formation phase around $t^{*}=3$, the vortex ring advances with the piston velocity (slope 1). For this Reynolds

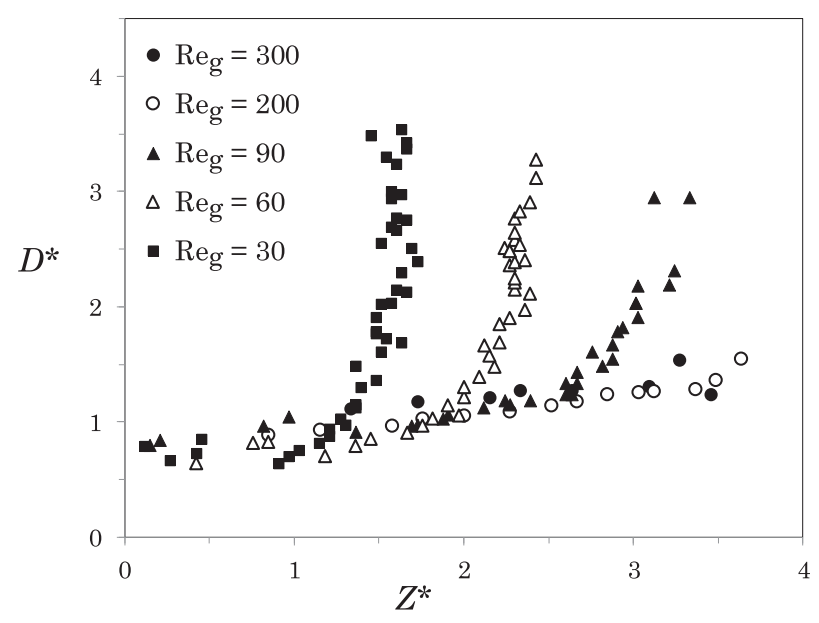

FIG. 9. Evolution of the vortex ring diameter as a function of its axial position in fluid X2 $(n=0.65)$ for different Reynolds numbers.

number, the flow has some inertia and this generation phase is followed by a propagation of the vortex ring at a similar speed close to half the piston velocity (slope 1/2), as classically observed at least in Newtonian fluids. ${ }^{20}$ After $t^{*} \sim 6$, viscous cross-diffusion alters the circulation of the vortex ring. For this reason, the vortex ring begins to decelerate in all cases but in a way which seems to differ with the power-law index.

This last trend is more noticeable at a lower Reynolds number of 30 [Fig. 8(b)], for which the last stage of the vortex ring evolution is visible in the measurement window. For this relatively low Reynolds number, the generation process is almost instantly followed by a deceleration phase after $t^{*} \sim 4$, whose rate is affected by the power-law index: the shearthinning nature of the fluid tends to slow down the vortex ring, which reaches shorter distances for decreasing values of $n$. Palacios-Morales and Zenit ${ }^{29}$ observed a similar trend in their experiments, namely, more important slowdown and shorter propagation distances for decreasing Reynolds numbers and/or power-law index. It should be noted that reducing the rheology of the different fluids solely to the power-law index may be misleading since the diffusion regime analyzed here coincides with a decay of the shear rate. The shear rate is expected to decrease eventually below the critical value $\dot{\gamma}_{0}$, and therefore, the subsequent dynamics of the vortex ring tends ultimately to become Newtonian in nature, with a constant viscosity corresponding to the value $\eta_{0}$ of the Newtonian plateau (see Sec. III E for a further discussion on this point).
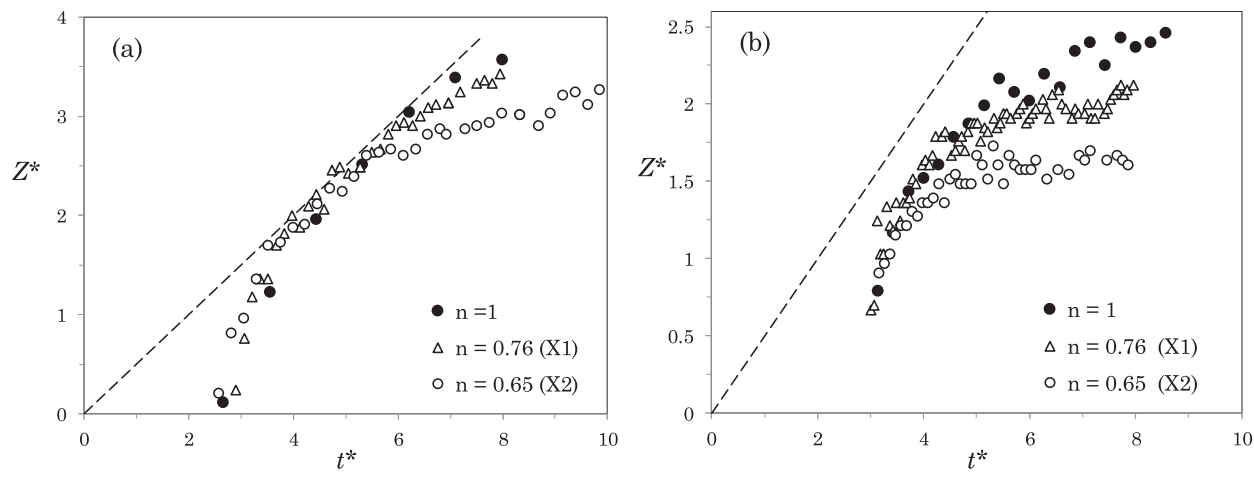

FIG. 8. Time evolution of the vortex ring position for different fluids at $R e$ $=R e_{g}=90$ (a) and $30(\mathrm{~b})$. 


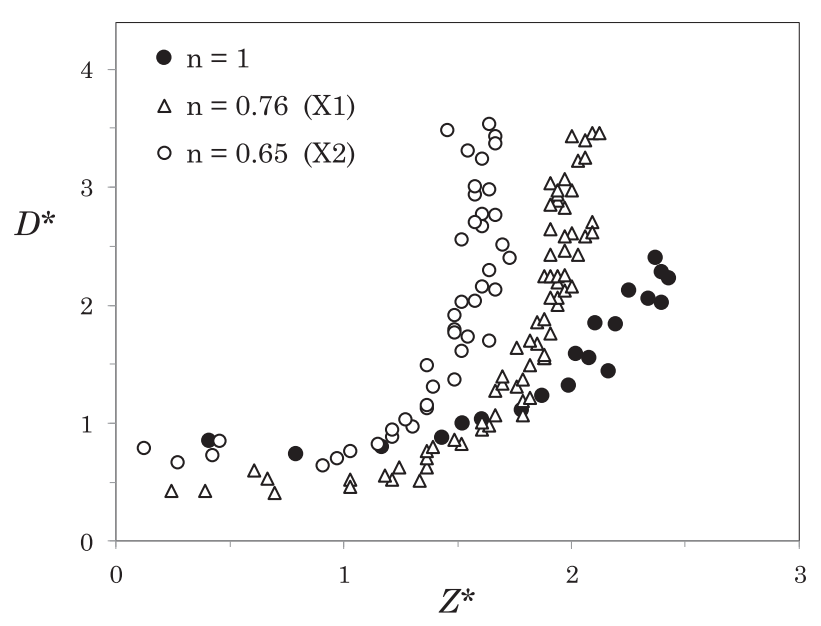

FIG. 10. Evolution of the vortex ring diameter as a function of its axial position for different fluids at $R e=R e_{g}=30$.

\section{Vortex ring size}

The evolution of the vortex ring diameter as a function of $z^{*}$ is displayed in Fig. 9 for the X2 fluid $(n=0.65)$ and for generalized Reynolds numbers from 300 down to 30 . In a first stage, the vortex ring diameter for all $R e$ is approximately constant around the same mean value close to the cylinder diameter. For the largest Reynolds numbers (200 and 300), the vortex ring diameter slowly increases but remains of the order of the cylinder diameter. At lower Reynolds number (90 and below), the evolution is radically different. The vortex ring undergoes a dramatic increase in its size as it slows down and eventually stops. This increase in diameter is more pronounced when the Reynolds number is lower, corresponding to shorter propagation distances.

The influence of the rheology on this phenomenon is illustrated in Fig. 10, which displays the evolution of the vortex ring diameter for different fluids for a Reynolds number of 30 . The initial stage of the vortex ring evolution shows no influence of the power-law index on the diameter, until $z^{*}=1$. By contrast, once the vortex ring has propagated over approximately one diameter, the evolution differs from one fluid to another. As previously, there is a direct correlation between the slowdown of the vortex ring and the increase in its diameter. A smaller power-law index seems to promote the growth of the vortex ring, which displays larger and quicker diameter growths for decreasing values of $n$ (as pointed out at the end of Sec. III C, recall however that reducing the rheology to the power-law index only may be misleading). It is noteworthy that Palacios-Morales and Zenit ${ }^{29}$ made similar observations in their experiments.

\section{E. Shear rate field}

With a view to deepening the analysis, the spatial distribution of the shear rate in the X2 fluid is displayed in Fig. 11 for different times at $R e_{g}=200,90$, and 30. Shear rate computation being based on velocity gradients, experimental errors from the PIV measurements are exacerbated, but we chose not to smooth out (nor symmetrize) the fields. This allows the level of error to be at least approximately inferred from the figures. The shear rate of transition between the Newtonian plateau and the shear-thinning behavior is $\dot{\gamma_{0}} \sim 0.5 \mathrm{~s}^{-1}$ (see Table I), and therefore the non-Newtonian regions are (a) $R e_{g}=200$
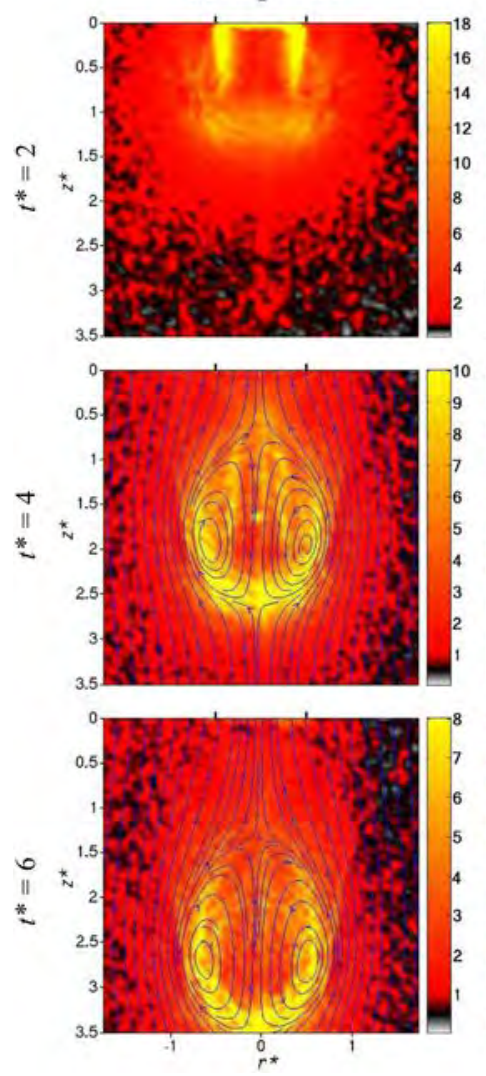

(b) $R e_{g}=90$

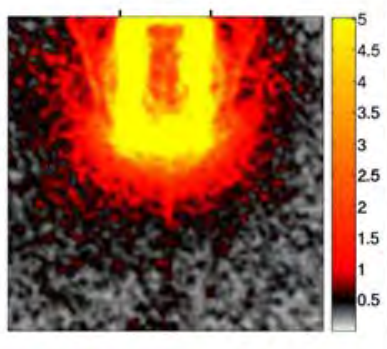

(c) $R e_{g}=30$

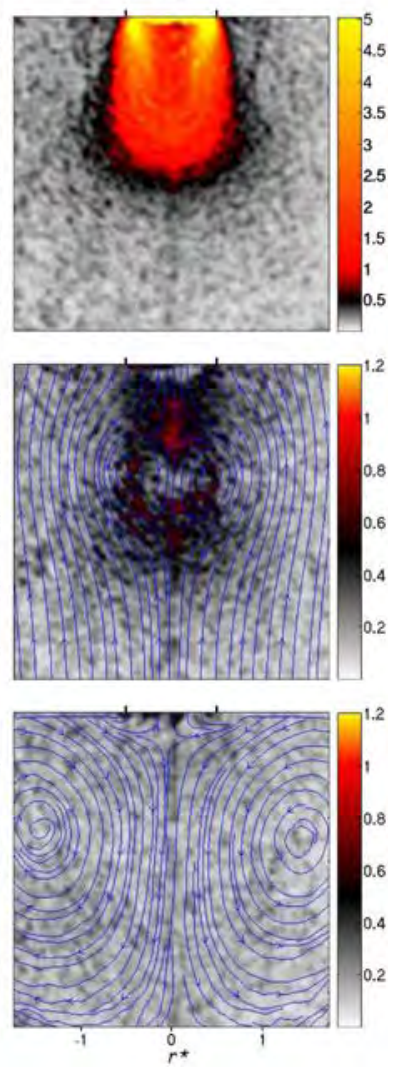

FIG. 11. Time evolution of the shear rate field and instantaneous streamlines in the moving frame of reference, for the $\mathrm{X} 2$ fluid $(n=0.65)$ at $R e_{g}=200$ (a), 90 (b), and 30 (c). 

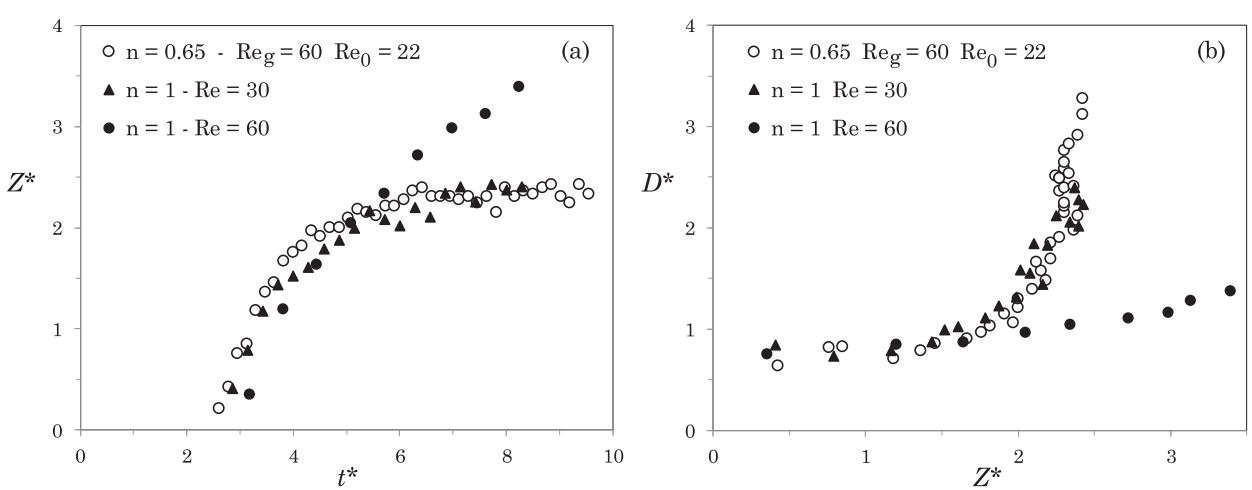

FIG. 12. Comparison of the evolution of the vortex ring position (a) and diameter (b) for the X2 fluid at $R e_{g}=60$ and in the Newtonian case at $R e=60$ and $\operatorname{Re}=30$. highlighted in red and yellow with the present colormap. The gray levels correspond to a Newtonian behavior associated with the constant viscosity plateau for shear rates lower than $\dot{\gamma_{0}}$. As observed in Figs. 11(a) and 11(b), the whole flow associated with the generation and propagation of the vortex ring at $R_{g}=200$ and 90 is subjected to shear-thinning, at least for the times displayed. This non-Newtonian behavior is initially the most pronounced in the shear layer emanating from the boundary layer detaching from the cylinder inner wall, before becoming progressively more prominent at the front of the developing vortex ring. By contrast, there is a minimum of shear rate on the axis between the vortex centers, where cross-diffusion of vorticity takes place.

For these large values of Reynolds number, the shearthinning behavior of the fluid and the subsequent large spatial variations of viscosity have no significant influence on the vortex ring propagation, whose speed can be qualitatively inferred from Figs. 11(a) and 11(b) to approximately half the piston velocity, and is therefore similar to the Newtonian reference case (see also Sec. III C). Indeed, even if viscosity displays strong variations in the vortex ring, the flow is dominated by inertia: since the propagation speed does not depend on viscosity for Newtonian vortex rings at large Reynolds numbers, it is consistent that the spatial variations of viscosity do not affect the vortex ring velocity for these inertia-dominated cases.

The scenario is radically different for $R e_{g}=30$. During the generation phase [see Fig. 11(c) for $t^{*}=2$ ], shear-thinning is present in the shear layer of the impulsive jet at the exit of the cylinder (which justifies the present choice of the generalized Reynolds number $R e_{g}$ as a control parameter for the vortex ring generation). But inertia is low at such a Reynolds number: the vortex ring instantly slows down, the shear rate rapidly decreases below the critical value $\dot{\gamma}_{0} \sim 0.5 \mathrm{~s}^{-1}$, and viscosity reaches the constant value $\eta_{0}$ associated with the Newtonian plateau. For $t^{*}=4$ and later, the whole flow therefore behaves as a Newtonian fluid. It is then expected that the last stage of the vortex ring before complete viscous dissipation is free of non-Newtonian effects and that the associated dynamics corresponds to a more relevant Reynolds number $R e_{0}=\rho V_{p} D_{p} / \eta_{0}$ which might be significantly different from the generalized Reynolds number $R e_{g}$ defined initially.

This conjecture has been confirmed by analyzing the endof-life dynamics of a vortex ring generated in the X2 fluid at a generalized Reynolds number of 60. Its trajectory after $t^{*} \sim 5$ is very different from that of a Newtonian vortex ring generated at the same Reynolds number [see Figs. 12(a) and 12(b)]. But if the Reynolds number of the non-Newtonian vortex ring is now defined with the viscosity corresponding to the Newtonian plateau (here $\operatorname{Re}_{0} \sim 22$ ), then the comparison with the evolution of a Newtonian vortex ring generated at a similar Reynolds number $(\operatorname{Re}=30)$ is much more adequate. This result illustrates the fact that the end-of-life of shearthinning vortex rings reaches ultimately a diffusion regime at a constant viscosity corresponding to the Newtonian plateau.

In that context, the influence of the rheology on the time evolution of the vortex ring position as displayed at $R e_{g}=30$ in Fig. 8(b), for instance, can now be interpreted more clearly. The shear rate of the X2 fluid is observed to fall below the critical value $\dot{\gamma}_{0}$ after $t^{*} \sim 4$ [Fig. 11(c)]. The same holds for the X1 fluid (data not shown). For this reason, the subsequent vortex ring evolution after this time is Newtonian in nature, at a constant viscosity $\eta_{0}$. At that stage, the generalized Reynolds number $R e_{g}$ used as a control parameter for the generation process is no more relevant and should be replaced by the Reynolds number $R e_{0}$ based on $\eta_{0}$. In the present case corresponding to $R e_{g}=30$, this Reynolds number $R e_{0}$ is equal to 23 and 14 for the X1 and X2 fluids, respectively. The difference between the decelerations observed in Fig. 8 is therefore due to the difference between the values of the Reynolds number $R e_{0}$, vortex rings diffusing and stalling sooner for lower $R e_{0}$.

\section{DISCUSSION AND CONCLUSIONS}

This study presents an experimental investigation of the dynamics of vortex rings generated by using a piston-cylinder device in shear-thinning fluids. PIV measurements and subsequent post-processing were performed in order to study the influence of the non-Newtonian behavior of the fluid. More particularly, experiments at low Reynolds number allowed for the experimental observation and physical analysis of the flow, from the generation phase up to the eventual dissipation of the vortex rings.

In the generation phase, the observations showed that shear-thinning controls the velocity profile of the jet at the cylinder exit. It is noteworthy that this result can be inferred from the Carreau number, which is always larger than unity in the present study. This justifies the present choice of the generalized Reynolds number $R_{g}$ (based on the solution for flows of power-law fluids in cylindrical ducts) as a relevant control parameter for the vortex ring generation. After the generation phase for large Reynolds numbers, vortex rings propagate due to self-induction: viscosity plays no determinant role in that 
process and, consistently, spatial gradients and shear-thinning do not influence the propagation either. Therefore, the propagation speed is independent of the Reynolds number and of the power-law index, at least as long as the regime is inertial. Because of vorticity cross-diffusion on the axis, the circulation of the vortex ring is expected to decrease progressively, leading the vortex ring to slow down. This phase is characterized by a global decrease in the shear rate which eventually falls below the critical value $\dot{\gamma}_{0}$ : the shear rate in this final stage ultimately reaches the Newtonian plateau, corresponding to a constant viscosity $\eta_{0}$. The whole flow therefore behaves as for a Newtonian fluid with this specific viscosity and with an effective Reynolds number $R e_{0}$ which is significantly lower than the initial generalized Reynolds number $R e_{g}$ used as a control parameter of the experiment. The results showed that the vortex ring end of life is indeed that of a Newtonian vortex ring at a Reynolds number based on the viscosity $\eta_{0}$ of the Newtonian plateau. This Reynolds number $R e_{0}=\rho V_{p} D_{p} / \eta_{0}$, which is known a priori, plays therefore the role of another control parameter in the description and analysis of the last, dissipative, phase of the vortex rings in shear-thinning fluids. Finally, the time at which the transition from shear-thinning to the Newtonian plateau occurs depends on the initial level of shear rate compared to the critical shear rate $\dot{\gamma}_{0}$. Therefore, the time and position of the slowdown and of the ultimate Newtonian dissipation phase are also controlled by the Carreau number.

\section{ACKNOWLEDGMENTS}

O.B. was supported by the Algerian government under PNE (Programme National d'Excellence), whose help is gratefully acknowledged.

\section{APPENDIX: SCALING LAW FOR THE VORTEX RING POSITION}

Following one referee's suggestion, we have tested a scaling of the form $Z^{*} R e_{g}^{m}$ for the time evolution of the vortex ring position displayed in Fig. 7. The best fit for the low Reynolds numbers 30, 60, and 90 using this change of variable was obtained for $m \sim-0.4$ (data not shown). This result cannot be linked to any obvious scaling law (like a viscous one, which would correspond to $m=-1$ ), and more importantly, it was not possible to find a satisfactory fit for all Reynolds numbers. This lack of a well-defined scaling law is probably due to the different physical regimes that should be taken into account: a propagation stage (which is expected to be mainly inertial and therefore Reynolds-independent) followed by a diffusion phase (which depends on the Reynolds number based on the viscosity of the Newtonian plateau). Therefore, this last stage depends also on the Carreau number, which compares a characteristic shear rate of the flow to the transition shear rate between the Newtonian plateau and the shear-thinning region. In this context, a scaling law for the position only and based solely on the Reynolds number is not expected to be found. A more complex form of the scaling law could be sought, but the present paper focuses on low Reynolds numbers (the final diffusive stage of the vortex rings at Reynolds numbers
200 and 300 is not quantitatively available with the experimental setup used in the present study), and this range of Reynolds numbers is certainly too limited in the hope of finding a robust and convincing scaling law.

${ }^{1}$ G. V. Lauder and E. G. Drucker, "Forces, fishes and fluids: Hydrodynamics mechanisms of aquatic locomotion," Physiology 17, 235-240 (2002).

${ }^{2}$ M. Gharib, E. Rambod, A. Kheradvar, D. J. Sahn, and J. O. Dabiri, "Optimal vortex formation as an index of cardiac health," Proc. Natl. Acad. Sci. U. S. A. 103, 6305-6308 (2006).

${ }^{3}$ Y. Bouremel, "On the circulation, strain, dynamics and scalar mixing in vortical flows," Ph.D. thesis, University of London, 2010.

${ }^{4}$ Y. Bouremel and A. Ducci, "Scalar mixing and strain dynamics methodologies for PIV/LIF measurements of vortex ring flows," Phys. Fluids 29, 013602 (2017).

${ }^{5}$ S. Benteboula, "Résolution des équations de Navier-Stokes à faible nombre de Mach: Application à l'étude de l'anneau de vorticité à masse volumique variable," Ph.D. thesis, University of Pierre and Marie Curie, Paris 6, France, 2006.

${ }^{6}$ D. An, A. Warning, K. G. Yancey, C. T. Chang, V. R. Kern, A. K. Datta, P. H. Steen, D. Luo, and M. Ma, "Mass production of shaped particles through vortex ring freezing," Nat. Commun. 7, 12401 (2016).

${ }^{7}$ T. Maxworthy, "The structure and stability of vortex," J. Fluid Mech. 51, 15-32 (1972).

${ }^{8}$ B. N. Didden, "On the formation of vortex rings: Rolling-up and production of circulation," Z. Angew. Math. Phys. ZAMP 30, 101-116 (1979).

${ }^{9}$ A. Dazin, P. Dupont, and M. Stanislas, "Experimental characterization of the instability of the vortex rings. Part I: Linear phase,” Exp. Fluids 40, 383-399 (2006).

${ }^{10}$ A. Dazin, P. Dupont, and M. Stanislas, "Experimental characterization of the instability of the vortex rings. Part II: Non-linear phase,” Exp. Fluids 41, 401-413 (2006).

${ }^{11} \mathrm{H}$. Helmholtz, "Über integral der hydrodynamischen gleichungen, welche den wirbelbewegungen entsprechen," J. Reine Angew. Math. 1858, 25-55.

${ }^{12}$ L. Kelvin, "The translatory velocity of a circular vortex ring," Philos. Mag. 33, 511-512 (1867).

${ }^{13}$ J. J. Thomson, A Treatise on the Motion of Vortex Rings (Macmillan, London, 1883).

${ }^{14} \mathrm{G}$. I. Taylor, "Formation of a vortex ring by giving an impulse to a circular disc and then dissolving it away," J. Appl. Phys. 24, 104-105 (1953).

${ }^{15} \mathrm{~T}$. Maxworthy, "The flow created by a sphere moving along the axis of a rotating, slightly-viscous fluid," J. Fluid Mech. 40, 453-479 (1970).

${ }^{16}$ M. H. I. Baird, T. Wairegi, and H. J. Loo, "Velocity and momentum of vortex rings in relation to formation parameters," Can. J. Chem. Eng. 55, 19-26 (1977).

${ }^{17}$ A. Glezer, "The formation of vortex rings," Phys. Fluids A 31, 3532-3542 (1988).

${ }^{18}$ K. Shariff and A. Leonard, "Vortex rings," Annu. Rev. Fluid Mech. 24, 235-279 (1992).

${ }^{19}$ J. J. Allen and B. Auvity, "Interaction of a vortex ring with a piston vortex," J. Fluid Mech. 465, 353-378 (2002).

${ }^{20}$ I. S. Sullivan, J. J. Niemela, R. E. Hershberger, D. Bolster, and R. J. Donnelly, "Dynamics of thin vortex rings," J. Fluid Mech. 609, 319-347 (2008).

${ }^{21}$ R. H. Hernandez and T. Reyes, "Symmetrical collision of multiple vortex rings," Phys. Fluids 29, 103604 (2017).

${ }^{22}$ M. Gharib, E. Rambod, and K. Sharif, "A universal time scale for vortex ring formation," J. Fluid Mech. 360, 121-140 (1998).

${ }^{23}$ S. E. Widnall, D. Bliss, and C. Y. Tsai, "The instability of short waves on a vortex ring," J. Fluid Mech. 66, 35-47 (1974).

${ }^{24}$ P. G. Saffman, "The number of waves on unstable vortex rings," J. Fluid Mech. 84, 625-639 (1978).

${ }^{25} \mathrm{~S}$. E. Widnall and C. Y. Tsai, "The instability of the thin vortex ring of constant vorticity," Philos. Trans. R. Soc., A 287, 273-305 (1977).

${ }^{26}$ P. G. Saffman, "Dynamics of vorticity," J. Fluid Mech. 106, 49-58 (1981).

${ }^{27}$ V. V. Meleshko, A. A. Gourjii, and T. S. Krasnopolskaya, "Vortex rings: History and state of the art," J. Math. Sci. 187(6), 772-808 (2012).

${ }^{28} \mathrm{O}$. Velasco Fuentes, "Early observations and experiments on ring vortices," Eur. J. Mech. B: Fluids 43, 166-171 (2014).

${ }^{29}$ C. Palacios-Morales and R. Zenit, "The formation of vortex rings in shearthinning liquids," J. Non-Newtonian Fluid Mech. 194, 1-13 (2013).

${ }^{30}$ P. M. Coelho and F. T. Pinho, "Vortex shedding in cylinder flow of shearthinning fluids: I. Identification and demarcation of flow regimes," J. NonNewtonian Fluid Mech. 110, 143-176 (2003). 
${ }^{31}$ A. Chandra and R. P. Chhabra, "Influence of power-law index on transitional Reynolds numbers for flow over a semi-circular cylinder," Appl. Math. Modell. 35, 5766-5785 (2011).

${ }^{32}$ M. K. Rao, A. K. Sahu, and R. P. Chhabra, "Effect of confinement on powerlaw fluid flow past a circular cylinder," Polym. Eng. Sci. 51, 2044-2065 (2011).

${ }^{33}$ A. K. Sahu, R. P. Chhabra, and V. Eswaran, “Two-dimensional unsteady laminar flow of a power law fluid across a square cylinder," J. Non-Newtonian Fluid Mech. 160, 157-167 (2009).

${ }^{34}$ A. K. Sahu, R. P. Chhabra, and V. Eswaran, "Two-dimensional laminar flow of a power-law fluid across a confined square cylinder," J. Non-Newtonian Fluid Mech. 165, 752-763 (2010).

${ }^{35} \mathrm{~S}$. Mishra and K. Jayaraman, "Asymmetric flows in planar symmetric channels with large expansion ratio," Int. J. Numer. Methods Fluids 38, 945-962 (2002).

${ }^{36}$ S. Dhinakaran, M. S. N. Oliveira, F. T. Pinho, and L. A. Alves, "Steady flow of power-law fluids in a 1:3 planar sudden expansion," J. Non-Newtonian Fluid Mech. 198, 48-58 (2013).
${ }^{37}$ N. Zhen, R. A. Handler, Q. Zhang, and C. Oeth, "Evolution of a hairpin vortex in a shear-thinning fluid governed by a power-law model," Phys. Fluids 25, 101703 (2013).

${ }^{38}$ K. Yasuda, R. C. Armstrong, and R. E. Cohen, "Shear flow properties of concentrated solutions of linear and star branched polystyrenes," Rheol. Acta 20, 163-178 (1981).

${ }^{39}$ P. R. de Souza Mendes, "Dimensionless non-Newtonian fluid mechanics," J. Non-Newtonian Fluid Mech. 147, 109-116 (2007).

${ }^{40}$ S. Shahsavari and G. H. McKinley, "Mobility of power-law and Carreau fluids through fibrous media," Phys. Rev. E 92, 063012 (2015).

${ }^{41}$ R. I. Tanner, Engineering Rheology (Clarendon Press, Oxford, 1988).

${ }^{42}$ C. Palacios-Morales and R. Zenit, "Vortex ring formation for low Re numbers," Acta Mech. 224, 383-397 (2013).

${ }^{43} \mathrm{~S}$. Maurel, "Etude par imagerie laser de la génération et de la rupture d'un écoulement tourbillonnaire compressé. Situation modèle pour la validation de simulations aux grandes échelles dans les moteurs," Ph.D. thesis, INP, Toulouse, France, 2000. 